\title{
Parallel tales of a changing brain
}

\author{
THE DYINC of \\ LIFE, DEATH, \\ end the \\ CHANGING \\ BRAIN

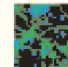 \\ The Dying of Enoch Wallace: Life, Death and the Changing Brain \\ by Ira B. Black \\ McGraw-Hill, New York, 2000. \$24.95 \\ hardcover, pp 260 \\ ISBN 0-07-136208-8 \\ IRA B. BLACK, M.D. \\ Reviewed by Bradley T. Hyman
}

Ira Black's new book is an intriguing scientific thriller that, like all good thrillers, weaves multiple plots. Black starts the book with a preface outlining what he hopes to accomplish. The ambition of the book is nothing short of drawing connections among mind, brain and molecules. He asks the rhetorical question, "How can we examine genes, brain, and mind in a single brief book? We experiment." Indeed, this book itself is an experiment, as it tells two parallel but related stories. In one, we follow the life of Enoch Wallace, a successful investment banker, as Alzheimer's disease "destroys his brain, his mind, and [his] humanity." In the second story, we learn about the discoveries of modern neuroscience that bear directly on Enoch's condition.

The parallel telling of clinical and scientific tales at its best breathes life, personality and clinical relevance into the neuroscience. Over the course of ten chapters, the main character in the clinical vignette goes from momentary distractibility to living in a nursing home. At the same time, the 'science thriller' develops the characters and creates caricatures of leaders in neuroscience in an ambitious, broad array of topics. It starts with LeviMontalcini and the discovery of nerve growth factor, touches on Trk receptors, cholinergic neuronal loss in Alzheimer's disease, the discovery that neurons could be cultured, and the discovery that growth factors represent many related families. From there, the science thriller goes on to apoptosis, stem cells, the role of glia and the conceptualization of memory from amnesia through LTP. The story about the role of glutamate in excitotoxicity, and the promise of neural transplantation contrast the sterile clinical approach of the neurol-

Bradley Hyman is at Harvard Medical School, Massachusetts General Hospital, Alzheimer Research Unit, 149 13th Street, Room 6405, Charlestown, Massachusetts 02129, USA.

e-mail:b_hyman@helix.mgh.harvard.edu ogist who documents Enoch Wallace's gradual deterioration.

The clinical vignettes are not limited to Alzheimer's disease. Stroke, Parkinson's disease, Huntington's disease, amyotrophic lateral sclerosis, neurosurgical lesions, the classic amnesic patient HM, and even alcoholism and dementia pugilistica are brought to bear to illustrate concepts of brain modularity and systems neuroscience. All this in 260 pages!

Nothing seems to delight Black more than reporting dogma that falls from grace. One of my favorite chapters is entitled "Brain Birthdays." The chapter starts with the continued deterioration of Enoch Wallace, continues with a brief clinical vignette on a Parkinson's disease patient, and then jumps into a wonderful ride about neuronal division and rebirth in the adult brain. Black starts out with, "The dogma has been quite simple: brain cells do not regenerate. Recovery after brain illness and injury does not occur." However, Black goes on to suggest that brain cells, like every other cell in the body, can divide-under the right circumstances.

We meet Pasko Rakic, whose initial studies suggested that neurons do not divide in the adult primate brain. However, contrasting data came from Fernando Nottebohm's laboratory with studies of the acquisition of bird song by canaries. Black is excited to tell the tale about how the bird brain has taught us so much, as the questions Nottebohm asked about bird song learning led far beyond a simple understanding of behavior. The male canary's learning of song depends on testosterone, which leads to dramatic changes in the brain system devoted to learning song. The brain structures increase by two- or threefold, partly as the result of the birth of new neurons. The excitement of the discoveries is palpable: "When they examined the brains they were dumbfounded...But neurons were not suppose to divide in adults."

Nottebohm and colleagues were left with the conclusion that neuronal division occurs in the canary brain. Black then follows up the idea of adult neuronal division, introducing us to many of the primary movers in the field. Neuronal reproduction in culture was finally established, and the birth of new neurons in rodents became established through the work of Bruce McEwen and Elizabeth Gould. The work proceeded rapidly to Old World monkeys. New techniques were applied: Rakic can now find neuronal birth in the adult Old World monkey brain. Rusty Gage and Anders Bjorklund pushed forward with neuronal transplantation, "foreshadowing entirely new approaches for treating brain disease." The chapter concludes with a vision of the future in which stem cells can be used for cell therapy in the brain for neurodegenerative diseases like Alzheimer's disease and Parkinson's disease.

This chapter can be viewed as a model for the entire book. Parallel stories are told, with clear inference that the influence of our expanding scientific understanding of the brain will have profound influences on clinical medicine. The dogma of 'neurons do not divide' fell to the power of simple, elegant experiments performed by scientist-heroes who went forward despite the common wisdom. The implication, and hope, not only of "Brain Birthdays," but of the entire book, is that the clinical dogma that Alzheimer's disease is incurable will also fall to simple, elegant experiments.

The narrative races along, and whereas the aficionado might complain of occasional minor mistakes or over-simplifications, the theme that the brain is a living, dynamic organ with potential for growth and plasticity comes through loudly and clearly. Unhappily, the final chapter in which all the different threads come together to a satisfying conclusion has yet to be written. The clinical problems are still separated from the neuroscientific advances by a considerable divide. But Ira Black's enthusiasm and belief that that divide is shrinking is contagious, and his enthusiasm and optimism make for a good read. 